\title{
Eigenmode multiplexing with SLM for volume holographic data storage
}

Guanghao Chen, Bo E. Miller, Yuzuru Takashima

Guanghao Chen, Bo E. Miller, Yuzuru Takashima, "Eigenmode multiplexing with SLM for volume holographic data storage," Proc. SPIE 10384, Optical Data Storage 2017: From New Materials to New Systems, 1038407 (23 August 2017); doi: 10.1117/12.2276985

SPIE Event: SPIE Optical Engineering + Applications, 2017, San Diego, California, United States 


\title{
Eigenmode Multiplexing with SLM for Volume Holographic Data Storage \\ Guanghao Chen, Bo E. Miller, Yuzuru Takashima* \\ University of Arizona, College of Optical Science \\ 1630 E. University Blvd., Tucson, AZ USA 85719
}

\begin{abstract}
The cavity supports the orthogonal reference beam families as its eigenmodes while enhancing the reference beam power. Such orthogonal eigenmodes are used as additional degree of freedom to multiplex data pages, consequently increase storage densities for volume Holographic Data Storage Systems (HDSS) when the maximum number of multiplexed data page is limited by geometrical factor. Image bearing holograms are multiplexed by orthogonal phase code multiplexing via Hermite-Gaussian eigenmodes in a $\mathrm{Fe}: \mathrm{LiNbO}_{3}$ medium with a $532 \mathrm{~nm}$ laser at multiple Bragg angles by using Liquid Crystal on Silicon (LCOS) spatial light modulators (SLMs) in reference arms. Total of nine holograms are recorded with three angular and three eigenmode.
\end{abstract}

Keywords: Holography, Data Storage, Spatial Light Modulator, Phase-code Multiplexing, Eigenmode Multiplexing

\section{INTRODUCTION}

In the current big data era enabled by rapid development of high speed communication and computer technology, the soaring data volume puts an alarming stress on the current storage systems, even though a large portion of the data ends up "frozen" in the data center. New storage structures and new storage technologies are on high demand to ease the impending storage crisis. Volume holographic data storage, which is energy efficient, and is capable of high storage density, and high stability, is a promising solution to relieve this "storage drought" [1].

The inherent nature of sensitive angular selectivity in volume holograms gives rises to a collection of Bragg-selectivity based multiplexing. Traditionally, multiplexing methods such as angular, wavelength, phase code, and speckle shifts have been used to increase the storage density [2]. In angular multiplexing, object and reference beams share common angular space, thus, maximum number of angular multiplexed holograms is geometrically limited. Recently, improvement of dynamic range, and reduction of material shrinkage allows us to multiplex hologram as close to as geometrical limit of the angular multiplexing [3,4]. A question is that if angular multiplexing approaches its geometrical limit, what the other multiplexing method can be combined with to fully use the material dynamic range. Among the multiplexing methods, orthogonal phase-code multiplexing requires no moving part and provides higher signal-to-noise ratio (SNR), factor of 2 compared to angular multiplexing [5].

We experimentally demonstrated that orthogonal phase code multiplexing by using eigenmode of cavity combined with angular multiplexing has affinity to existing angular multiplexing while optically enhancing dynamic range and/or sensitivity of material [6].

In our research, Hermite-Gaussian eigenmodes are adopted, since they can be further enhanced with a resonant cavity. Conventionally, phase plates are adopted to generate orthogonal modes [6]. Eigenmode multiplexing with static phase plates is reported to yield a crosstalk as low as $2.5 \%$. However, the use of static phase plates requires mechanical movement to toggle phase patterns. To implement non-moving part eigenmode multiplexing, electronically addressed liquid crystal based spatial light modulator (LC-SLM) is a preferable choice. In addition, SLMs have a short response time, which greatly accelerates the recording and readout process as compared to mechanically toggling phase patterns. Nonetheless, in contrast to static phase plates, LC-SLM are less stable and accurate in terms of phase modulation, which greatly reduces their use in precise applications. As far as we know, research on using SLM to dynamically alter the eigenmode the reference beam in HDS has not yet been reported.

In the following context, our research on volume hologram multiplexing with eigenmodes converted with LC-SLM is introduced. First, we will briefly introduce principle phase-coded multiplexing. Then, the experimental setup and

\footnotetext{
*ytakashima@optics.arizona.edu
} 
experimental procedures are introduced. In discussion section and conclusion section, results are analyzed and a conclusion is made on the base of the analysis.

\section{THEORY}

Reconstructed field by phase-code multiplexing method is described as the cross-correlation function of the writing reference beam and reconstructing probe beam. The cross-correlation of the arbitrary complex field $\mathrm{h}$ and $\mathrm{g}$ in grating space can be expressed as follows [6].

$$
(g \otimes h)(\vec{k})=\int_{\mathbb{R}^{3}} g^{*}\left(\overrightarrow{k^{\prime}}-\vec{k}\right) h(\vec{k}) d \vec{k} .
$$

For orthogonal functions indexed by two mode numbers, the following relation is satisfied.

$$
\left\langle g_{n, m} \mid g_{p, q}\right\rangle(\vec{k})=\int_{\mathbb{R}^{3}} g_{n, m}(\vec{k}) g_{p, q}^{*}(\vec{k}) d \vec{k}=\delta_{n, p} \delta_{m, q}
$$

where

$$
\delta_{n, m}= \begin{cases}0, & n \neq m \\ 1, & n=m\end{cases}
$$

Hence, theoretically, the correlation coefficients between fields that are described by a collection of orthogonal functions will be zero and thus it is possible to multiplex holograms with orthogonal beams with low crosstalk.

\section{EXPERIMENTS}

At the reference arm, a phase-only Liquid Crystal on Silicon (LCoS) SLM (0.7-inch SXGA+, JVC, Japan) extracted from a commercial projector (Canon Model SX50) is incorporated by a $90^{\circ}$ corner cube geometry to fold the beam path to the SLM (Figure 1). The SLM has an 8-bit gray scale corresponds to 0 to $\pi$ phase modulation at $532 \mathrm{~nm}$ (shown in the phase detection result in Figure 1) and the resolution of the SLM is 2500 pixels per inch.

The SLM was placed outside the Rayleigh range of the incident beam to perform Fourier transform of the phase pattern on the SLM. On the SLM, the phase of the reflected light is modulated by the phase retardance of the pixel where the light hits. Gray levels $0 \sim 255$ are mapped to $0 \sim \pi$ phase retardance. Gray level 0 (color black) indicates no phase modulation on the corresponding pixels, while 255 (color white) indicates $\pi$ phase retardance on the corresponding pixels. Phase detection result is shown in inset of Fig. 1. Fringes in the central region are shifted half wave, corresponding to the $\pi$ phase retardance of the SLM. The +1 order was used to interfere with the signal beam in a $10 \times 10 \times 20 \mathrm{~mm} \mathrm{Fe:} \mathrm{LiNbO}_{3}$ crystal (Deltronic Crystal Industries) while the other diffraction orders are blocked by the iris. More details of this part will be explained later in this paper. A $\lambda / 2$ plate matches the polarization of the object and reference beams. In the reference path a $\mathrm{f}=50 \mathrm{~mm}$ lens inserted between the iris and the crystal scales the size of reference beam so that the sizes of the object and reference beams in the crystal are comparable. The beam waist of the reference beam is located at the center of the recording medium.

This setup uses Fourier-plane recording geometry. At the signal arm, the Gaussian beam is expanded and illuminates a USAF target plate. A microscope objective (Model 80.3020, Rolyn Optics) is used to perform Fourier transform of the signal. Pattern of spatial frequencies is located at the beam waist of the transformed beam, intersecting the reference arm at the center of the crystal. An additional camera was placed after crystal to capture the signal, measure the normalized crosstalk of the experiment. 


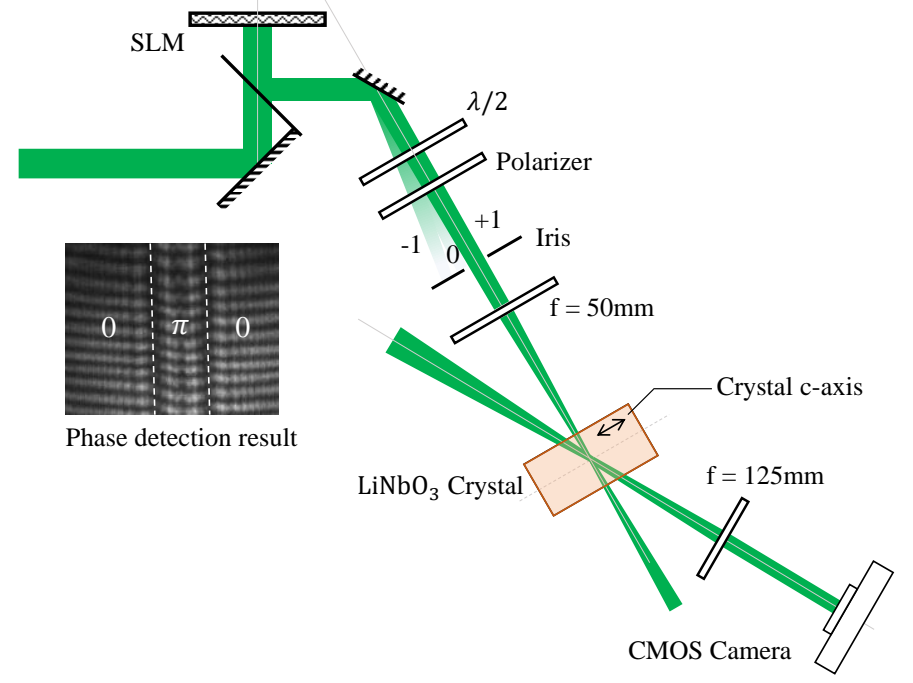

Figure 1. Experimental setup. Inset: phase detection result

As is shown in Fig. 2, by comparing the valley of the intensity profiles, the mode conversion result of static phase plate in (a) is closer to the ideal $\mathrm{HG}_{10}$ mode than the result in (b). The latter was converted by the SLM. The shallow valley in (b) indicates that there is a substantial mode crosstalk.

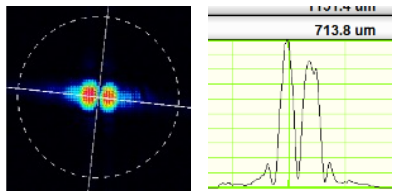

(a)

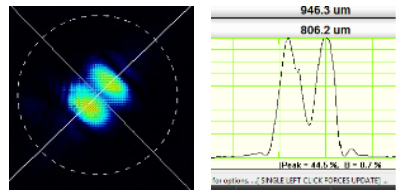

(b)

Figure 2. Comparison of phase plate and SLM HG 10 mode conversion results. (a) is the mode conversion result of static phase plate and (b) is that of the LCoS SLM. In terms of intensity profile, mode in (a) is closer to pure $\mathrm{HG}_{10}$ mode. The shallow valley on the profile in (b) indicates that there is severe mode crosstalk.

This problem is partly due to the reflection of light from the surfaces in each optical element. To improve purity of modes, in our experiments, we added a square-wave grating phase on top of the original phase patterns. The grating phase can generate multiple diffraction orders, as we mentioned in the setup section, and avoid the reflections along the original beam path. Figure 3. Phase maps and mode conversion results. The first row shows the phase patterns used to generate different Hermite-Gaussian modes. The second row shows the phase patterns after a grating phase is added. The third row shows the mode conversion results. Color red denotes the peak of the intensity. (b) shows an improved result with grating phase added. shows phase profiles on SLM and corresponding intensity profile. Three phase profiles, $\mathrm{HG}_{00}, \mathrm{HG}_{10}$ and $\mathrm{HG}_{20}$ are displayed on SLM. For $\mathrm{HG}_{20}$, the center portion of SLM has a width $\mathrm{d}$. The beam profile of the improved result is shown in (b). 

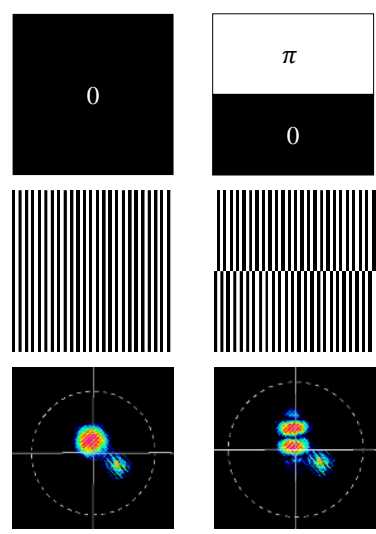

(a)

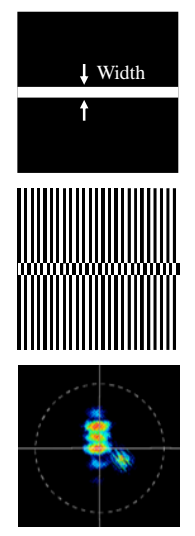

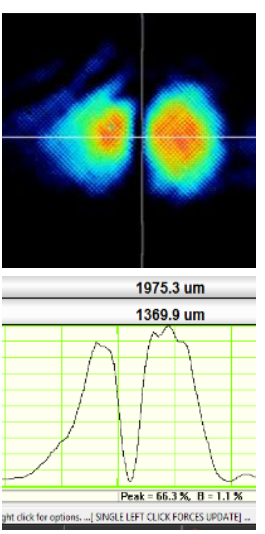

(b)

Figure 3. Phase maps and mode conversion results. The first row shows the phase patterns used to generate different HermiteGaussian modes. The second row shows the phase patterns after a grating phase is added. The third row shows the mode conversion results. Color red denotes the peak of the intensity. (b) shows an improved result with grating phase added.

As the grating period increases, diffraction efficiency of +1 and -1 orders increases, however, the diffraction angle decreases. In our experiment, a grating period of 4 pixels $(41.6 \mathrm{um})$ produced $31 \%$ diffraction efficiency of the grating. Relevant work done by Márquez et al. shows that the low diffraction efficiency is due to the loss of phase and is related to the period of the grating [7]. Mode conversion results are monitored by the beam profiler (WinCamD-UCD23, DataRay).

In generating $\mathrm{HG}_{20}$ mode, the width of the central region was optimized to reduce crosstalk between modes. To determine the optimum width of this region, we evaluated cross talk as a function of the central width of the $\pi$ region that can yield the lowest crosstalk. Firstly, a hologram was written with $\mathrm{HG}_{00}$ beam. Then, the hologram is constructed by $\mathrm{HG}_{20}$ beams with a different central region width. A CMOS camera with fixed exposure time is used to measure the diffracted power. Finally, we can plot a curve that indicates the setting where the least crosstalk occurs, as shown in Figure 4.

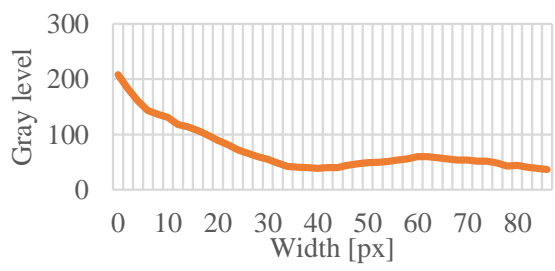

Figure 4. Intensity curve of hologram written with $\mathrm{HG}_{00}$ beam and read with $\mathrm{HG}_{20}$ beam. This curve was measured with increasing width. The minima of the curves are located at around 44 pixels; however, we can observe intensity drops as the width increases over 60 pixels.

The reading process bleached the hologram gradually. We can expect a decrease in diffracted power and inaccurate reading. To address this data bias, measurement with reversed steps were conducted, shown in Figure 5. Similarly, we wrote the hologram with $\mathrm{HG}_{10}$ and then read it by $\mathrm{HG}_{20}$ modes. The lowest crosstalk for both measurement occurs at width of 44 pixels (457.6 um). This setting was used in our further recording experiments. 


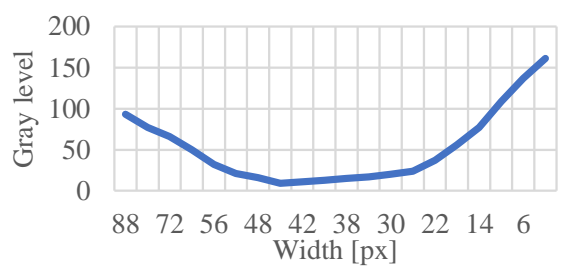

Figure 5. Reverse measurement with decreasing steps. Local minima can be found at 44 pixels, which confirms the result in Figure 4.

\section{RESULT ANALYSIS AND DISCUSSIONS}

In this experiment, a total of nine holograms are multiplexed by using three orthogonal modes at three different angles. Recording results are shown as follows (Figure 6). In experiments, a $0.6^{\circ}$ angle increment is used[6]. The images in the dotted lines are the objects, indicating the locations.

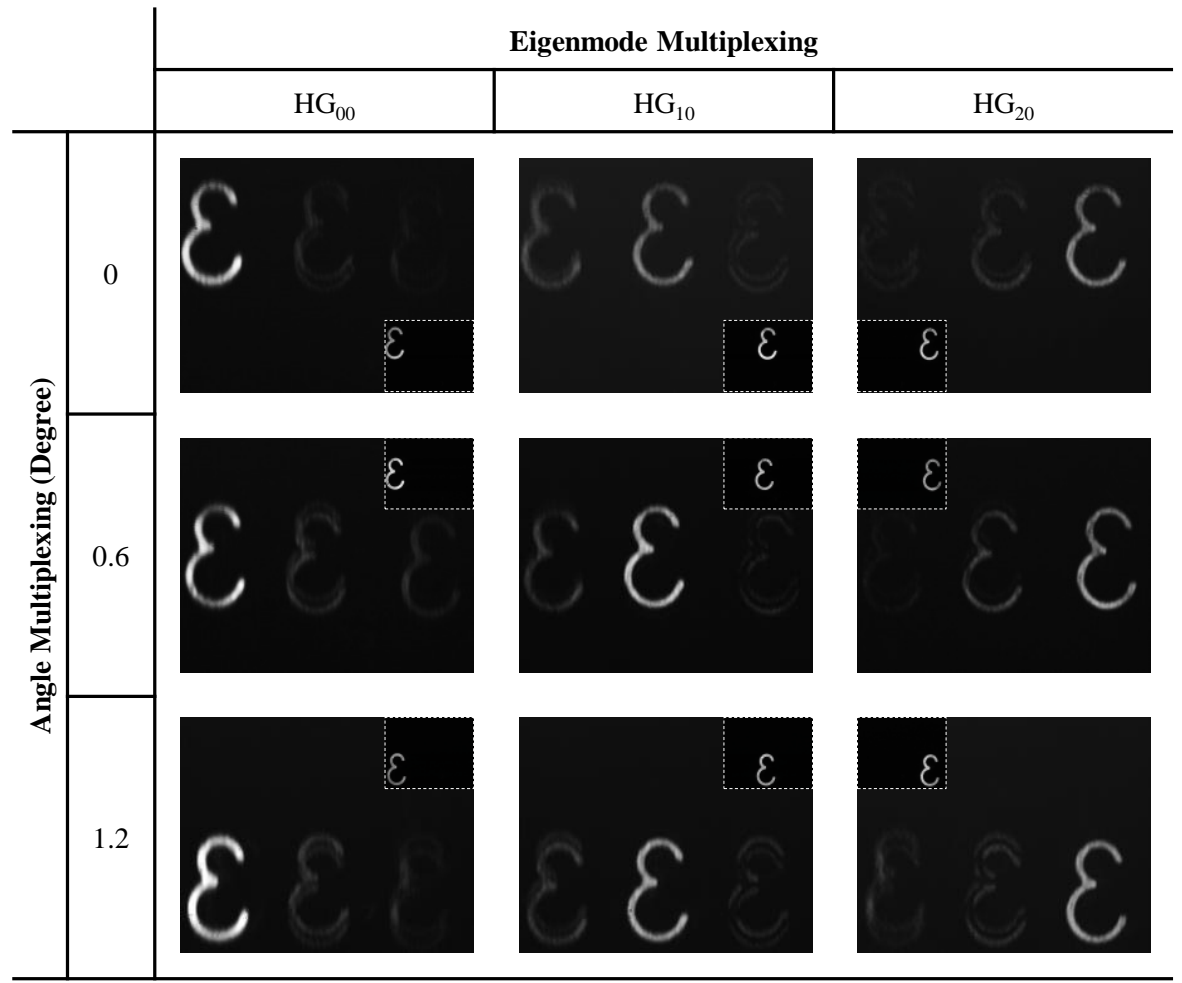

*Image brightness is increased due to low diffraction efficiency. Images shown inside the dash lines are the original signal

Figure 6. Nine holograms are recorded and reconstructed with eigenmode multiplexing and angle multiplexing.

As we can see in Figure 6. Nine holograms are recorded and reconstructed with eigenmode multiplexing and angle multiplexing., the implementation of SLM can still maintain a reasonable recording quality in the experiment where high frequencies can be captured and reconstructed. However, compared to phase-coded multiplexing with static phase plates, noticeable crosstalk between modes was observed. Crosstalk measurement results are shown in Table 1, where around 30\% crosstalk is observed. Besides, with SLM, eigenmode multiplexing crosstalk is more obvious than angular multiplexing crosstalk. 
Table 1. Mode Crosstalk

\begin{tabular}{|c|c|c|c|c|c|c|c|c|c|c|}
\hline & \multicolumn{3}{|c|}{$\mathrm{HG}_{00}$ Mode Crosstalk } & \multicolumn{3}{|c|}{$\mathrm{HG}_{10}$ Mode Crosstalk } & \multicolumn{3}{|c|}{$\mathrm{HG}_{20}$ Mode Crosstalk } \\
\hline & & $\mathrm{HG}_{00}$ & $\mathrm{HG}_{10}$ & $\mathrm{HG}_{20}$ & $\mathrm{HG}_{00}$ & $\mathrm{HG}_{10}$ & $\mathrm{HG}_{20}$ & $\mathrm{HG}_{00}$ & $\mathrm{HG}_{10}$ & $\mathrm{HG}_{20}$ \\
\hline \multirow{3}{*}{ 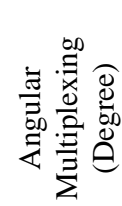 } & 0 & 100 & 21.7126 & 16.5575 & 25.5032 & 100 & 28.4646 & 37.7885 & 34.0900 & 100 \\
\hline & 0.6 & 12.5535 & 12.8236 & 12.6361 & 17.5574 & 16.4608 & 14.3429 & 26.1693 & 26.7866 & 27.3231 \\
\hline & 1.2 & 11.5000 & 11.5510 & 11.5059 & 13.5750 & 13.5167 & 13.3955 & 21.8659 & 23.9334 & 25.7227 \\
\hline
\end{tabular}

he high crosstalk in the experiment originates would be attributed to a lack of orthogonality between modes. Also, the sizes of the reference beam and signal beam account for some crosstalk. At the beam intersection point in the medium, to capture most of the frequency information of the object, the reference beam should be slightly larger than the signal beam, but it shouldn't be much larger considering the power density of the beams with given input power. In that case, if the $\mathrm{HG}_{00}$ mode is well calibrated, as the mode number increases, the size of the reference beam will inevitably be even larger than the signal beam. The mismatch of the sizes will reduce the orthogonality of fields and thus the condition in Eq. (3) is no longer satisfied. Another factor is that the phase patterns are not calibrated and thus these eigenmodes are not completely orthogonal to each other. At last, according to [8], factors including loss of phase modulation depth, phase linearity and insufficient spatial resolution of the SLM can lead to phase distortion, which impairs the orthogonality of the modes. The fly-back phenomenon in liquid crystal pane can substantially distort the phase patterns. In other words, the panel is a lowpass filter where high frequency phase variations will not pass. The $31 \%$ diffraction efficiency ( $40.5 \%$ in theory) of the square-wave grating indicates that phase loss occurs. Simulations and measurements should be carried out to further validate the orthogonality of eigenmodes and quantify the crosstalk. Cavity eigenmode enhancement can be applied to lower the crosstalk.

\section{CONCLUSIONS}

In this paper, research on dynamic reference beam modulation with phase-only LCoS SLM was demonstrated. A series of improvements, including reflection reduction by adding grating phase and phase pattern calibration, are brought in to enhance the performance of the SLM. Recording experiment with angular multiplexing and eigenmode multiplexing methods are conducted, recording nine holograms in total. On average, 30\% mode crosstalk is observed. Despite the high crosstalk, the implementation of SLM in reference beam phase encoding is considered feasible, given that a nonprofessional SLM and uncalibrated phase patterns are used. Further simulation and measurements should be carried out to address the crosstalk issue.

\section{REFERENCE}

[1] H. H. J. Coufal, G. G. T. Sincerbox, D. Psaltis, and G. G. T. Sincerbox, Holographic Data Storage. 2000.

[2] K. Curtis, L. Dhar, A. Hill, W. Wilson, and M. Ayres, Holographic data storage : from theory to practical systems. 2010.

[3] T. Tanaka and S. Kawata, "Comparison of recording densities in three-dimensional optical storage systems : multilayered bit recording versus angularly," J. Opt. Soc. Am. A, vol. 13, no. 5, pp. 935-943, May 1996.

[4] F. Askham, M. R. Ayres, and A. C. Urness, "High dynamic range holographic data storage media," Proc. SPIE, vol. 9587. pp. 958706-958708, 2015.

[5] L. Hesselink, S. S. Orlov, and M. C. Bashaw, "Holographic data storage systems," in Proceedings of the IEEE, 2004, vol. 92, no. 8, pp. 1231-1280.

[6] B. E. Miller and Y. Takashima, "Cavity-enhanced eigenmode and angular hybrid multiplexing in holographic data storage systems," Opt. Express, vol. 24, no. 26, p. 29465, Dec. 2016.

[7] A. Marquez, F. J. Martinez, R. Fernandez, S. Gallego, S. Fenoll, M. Ortuno, C. Neipp, I. Pascual, and A. Belendez, "Effective modeling of PA-LCoS devices and application in data storage in photopolymers," in 201615 th Workshop on Information Optics (WIO), 2016, pp. 1-3.

[8] I. Moreno, C. Iemmi, A. Márquez, J. Campos, and M. J. Yzuel, "Modulation light efficiency of diffractive lenses displayed in a restricted phase-mostly modulation display," Appl. Opt., vol. 43, no. 34, p. 6278, Dec. 2004. 